\title{
SENADOS, REPRESENTACIÓN POLÍTICA Y REPRESENTACIÓN TERRITORIAL: REFLEXIONES SOBRE UNA EXTENDIDA CONFUSIÓN
}

ROBERTO L. BLANCO VALDÉS ${ }^{\dagger}$

\section{LOS ORÍGENES: BICAMERALISMO SOCIAL Y BICAMERALISMO FEDERAL}

El bicameralismo ha presentado, a lo largo de la evolución del constitucionalismo, una naturaleza política compleja, como consecuencias sobre todo del hecho de que no ha habido históricamente, en realidad, un bicameralismo sino dos: el social y el federal. El bicameralismo originario -el social-, en el que cada una de las cámaras del parlamento representaba a distintos sectores de la sociedad, nació en realidad con mucha anterioridad al federal, cuando, a mediado del siglo XIV se creó en Inglaterra la Cámara de los Comunes, que, «representativa de las villas y condados en que estaba dividido el reino», se unió a la Cámara de los Lores, que «personificaba a los estamentos privilegiados» de la sociedad inglesa de la época ${ }^{1}$. Asentados en el parlamento de Westminster a partir de 1341, los Comunes no consiguieron, en todo caso, equiparar su poder al de los Lores hasta mediados del siglo XVII, cuando, tras el reinado de Isabel (1558-1603) y durante todo el período que acabaría por conducir a la Primera Guerra Civil (1642-1646) se culminó en Inglaterra el proceso que Lawrence Stone ${ }^{2}$ ha calificado como el de «la crisis de la aristocracia». Sin embargo, y pese al creciente poder que fue ganando en Gran Bretaña la Cámara de los Comunes a medida que, ya a partir del siglo XVIII, avanzaba el proceso de progresiva parlamentarización de su monarquía constitucional, la Cámara de los Lores conseguiría no sólo pervivir sino además convertirse en un auténtico modelo a imitar en toda la Europa continental, donde su existencia sirvió para impulsar la generalización del bicameralismo social a partir sobre todo de la restauración monárquica que siguió a la derrota de Napoleón y a la reunión del Congreso de Viena entre 1814 y 1815. De este modo, frente al monocameralismo que caracterizó a las

\footnotetext{
+ Catedrático de Derecho Constitucional Universidad de Santiago.

${ }^{1}$ Miguel Artola, Constitucionalismo en la historia, Barcelona, Crítica, 2005, p. 118.

2 Lawrence Stone, La crisis de la aristocracia. 1558-1641, Madrid, Alianza Editorial, 1985.
} 


\section{SENADOS, Representación Política y RePRESENTACión SOCIAL}

Constituciones revolucionarias (las francesas de 1791 y 1793, la española de 1812 o la portuguesa de 1822), los parlamentos configurados en las normas supremas de las monarquías constitucionales que dominaron la vida europea en el período que media entre la segunda década del siglo XIX y su crisis a partir del último tercio de la centuria serán, casi sin excepciones, bicamerales ${ }^{3}$. Vendrán a convivir así en Europa una cámara baja de representación popular y electiva -aunque designada durante décadas por sufragio censitario y/o capacitario- y una cámara alta (denominada generalmente Senado) cuya finalidad habrá de ser descrita con una meridiana claridad por el diputado moderado François-Antoine Boissy de Anglas, quien, tras el golpe de Estado de termidor que puso fin al período álgido de la Revolución Francesa, justificaría la inclusión de una segunda cámara parlamentaria en la Constitución francesa de 1795 echando mano de una, muy expresiva, y bellísima metáfora: con ella, se trataba, proclamará $\mathrm{D}^{\prime}$ Anglas, de que la razón corrigiese a la imaginación de la República ${ }^{4}$ Superadas ya las condiciones históricas que hicieron posible en Europa ese bicameralismo social, la existencia de parlamentos con dos cámaras ha pervivido modernamente, pese a todo, no sólo en su modelo federal. Así lo demuestran algunos sistemas de bicameralismo funcional (el italiano, por ejemplo) en los que las dos cámaras no se distinguen por su carácter social (una representativa y otra aristocrática u oligárquica como en la Europa del siglo XIX) sino por una diversa naturaleza funcional que viene dada por los diversos procedimientos de elección de una y otra o por sus respectivas competencias: tal ha acontecido, también, en algunos de los países del este de Europa que accedieron a la democracia tras la caída del Muro de Berlín 5 .

${ }^{3}$ Me he referido con detalle a todo este proceso histórico en el capítulo octavo de mi libro La construcción de la libertad. Apuntes para una historia del constitucionalismo europeo, Madrid, Alianza Editorial 2010, pp. 171-211.

4 «El Consejo de los Quinientos [cámara baja] compuesto de miembros más jóvenes, propondrá los decretos que crea útiles y se constituirá en el pensamiento y, por decirlo así, en la imaginación de la República; el Consejo de los Ancianos [cámara baja] constituirá la razón, no tendrá otra función que examinar con sabiduría cuales serán las leyes a admitir y rechazar, sin poder jamás realizar proposiciones», en Moniteur Universel, reimpresión, tomo XXV, pp. 81 y ss., cit. en M. Deslandres, Histoire constitutionnelle de la France de 1789 à 1870, Tomo I: De la fin de l'Ancien Régime à la chute de l'Empire, París, Armand Colin, 1932, p. 320.

${ }^{5}$ Aparte de los dos supuestos de bicameralismo federal (Rusia y la República de BosniaHerzegovina) regulan un sistema parlamentario bicameral de tipo funcional, en el que las dos Cámaras representan lo mismo y sólo se distinguen por su posición relativa en 
Frente a este bicameralismo social, de largo recorrido histórico durante el siglo XIX, el bicameralismo federal tendrá unos orígenes y una evolución muy diferentes. Su nacimiento puede datarse sin problemas, pues surge con la Constitución norteamericana, cuyo Congreso se compondrá de una asamblea de representación popular (la Cámara de Representantes) y de otra de representación territorial (el Senado), cámara esta última cuya naturaleza vendrá determinado, de forma primordial, por más que no sin un profundo enfrentamiento en los momentos del debate constituyente de Filadelfia, por la paridad de miembros que la conforman: dos por cada uno de los estados de la Unión. Ese modelo de Senado de tipo federal tardará sin embargo en extenderse, pues no será hasta la aprobación de la Constitución Suiza de 1848 cuando volvamos a encontrarlo: ciertamente, la Asamblea Legislativa federal del país alpino estaba entonces constituida por un Consejo Nacional, compuesto por diputados elegidos por la población en proporción a la de los cantones, y una Consejo de Estado, formado por 44 diputados, a razón de dos por cada cantón, dado que el país lo componían entonces un total de 22 territorios cantonales 6 . La mímesis con el modelo de los Estados Unidos resultaba, por tanto, a este respecto, indiscutible. Pero el modelo se extenderá con posterioridad: en efecto, a partir de mediados del siglo XIX la práctica totalidad de los parlamentos de los Estados federales que irán apareciendo en diferentes partes del planeta serán bicamerales, aunque las coincidencias resultarán menores no sólo en aspectos adjetivos (por ejemplo, la denominación de cada cámara), sino también en los más claramente sustantivos, es decir, en todos los relacionados con su configuración y sus funciones. Esa, la de la generalización, es la razón fundamental por la que la definición del federalismo como forma de Estado se ha ligado a la existencia de un sistema (real o presunto) de bicameralismo federal. Real o presunto, sí, porque la referida variedad,

el ejercicio de las funciones legislativa y de control (bicameralismo perfecto o imperfecto) o por su forma de elección (formula mayoritaria o proporcional; edad para poder ejercer el sufragio pasivo) la República Checa, Rumania y Polonia. Un caso especial es el de Eslovenia., en donde junto a una Asamblea Nacional, elegida por sufragio universal, existe un Consejo Nacional, de naturaleza corporativa, formado por representantes de los empresarios, trabajadores, agricultores, artesanos, profesionales; y por representantes de las actividades no económicas y de los intereses locales. En Croacia, en fin, la Cámara de los Condados prevista en la ley fundamental fue eliminada en la reforma constitucional de 2001. Todos los demás Estados tienen un parlamento unicameral, incluida Serbia, pese a su sistema autonómico.

${ }^{6}$ La Constitución suiza de 1848 puede verse, en su versión original, en Digithèque de matériaux juridiques et politiques (http://mjp.univ-perp.fr/constit/constitintro.htm). 


\section{SENADOS, Representación Política y RePRESENTACión SOCIAL}

aunque no sólo ella, obligan a revisar la tesis de que allí donde existe una segunda cámara que por su composición y/o su forma de elección presenta, en mayor o menor grado, naturaleza territorial estemos en presencia de una cámara que, a diferencia de la del pueblo, es expresión (o eso, al menos, se proclama) de la voluntad de los territorios federados. Tal revisión constituye el objeto primordial de las páginas que siguen

\section{DESCRIPCIÓN DE LOS DENOMINADOS SENADOS FEDERALES}

Tal presunción, que se afirma habitualmente como un parti pris que, por serlo, no necesita ulterior demostración, no responde, a mi juicio, a la realidad o, por lo menos, no en la medida en que se afirma. Antes, de todos modos, de abordar esa cuestión, expondré, con la mayor brevedad que sea posible, el estatuto (forma de composición, de elección y de actuación) de las distintas cámaras territoriales de los más importantes Estados federales del planeta, para tratar luego de elucidar si son o no en realidad territoriales, tal y como se afirma de forma casi general. Pues sólo si lo fueran sus funciones contribuirían a la integración federal que de ellas se predica.

Los Padres Fundadores de la nación americana no dudaban, desde luego, de que la creación de dos cámaras en su parlamento nacional respondía a la finalidad de representar a los electores según dos principios diferentes, y ello por más que la propuesta de un sistema de representación paritaria en el Senado diera lugar a una dura controversia que sólo pudo resolverse finalmente tras la firma del llamado Compromiso de Connecticut ${ }^{7}$. La idea iba a quedar clara, desde luego en los comentarios de El Federalista, en los que James Madison la apuntará con manifiesta transparencia: la Cámara de Representantes -escribe el futuro presidente de la Unión-, «derivará sus poderes del pueblo de América», pues «el pueblo estará representado en la misma en proporción y con arreglo al mismo principio que en la legislatura de un estado particular. Hasta aquí el gobierno es nacional y no federal». Sin embargo, y en contraposición con este principio de predominio de lo nacional sobre lo federal, se alzará el Senado, que «recibirá su poder de los estados, como sociedades políticas y coiguales», que "estarán representadas en el Senado conforme al principio de igualdad, como lo están ahora en el actual Congreso. Hasta aquí el gobierno es federal y no

\footnotetext{
${ }^{7}$ Cfr., al respecto, William H. Riker, «The Senate and the American federalism», en William H. Riker, The Development of the American Federalism, Boston, Kluwer Academic Publishers,1987, pp. 135-156.
} 
nacional». ${ }^{8}$ La igualdad de representación estatal en el Senado que, en mucha mayor medida que la designación de los senadores por las legislaturas estatales, constituirá, pues, el elemento condicionador fundamental del carácter territorial de la segunda cámara del Congreso de la Unión, se justificará como un elemento de transacción y de concordia entre los estados más pequeños y más grandes, pero esa forma primordial de argumentar no iba a impedir al propio Madison poner de relieve la doble utilidad de la nueva cámara. En primer lugar, como elemento de integración política en un Estado compuesto: «Si innegablemente es lo debido, en el caso de un pueblo fundido completamente en una sola nación, que cada distrito participe proporcionalmente en el gobierno y, en el caso de Estados independientes y soberanos, unidos entre sí por una simple liga, que las partes pese a la desigualdad de su extensión, tengan una participación igual en las asambleas comunes, no parece carecer de razón que en una república compuesta, que participa a la vez del carácter federal y del nacional, el gobierno se funde en una combinación de los principios de la igualdad y la proporcionalidad de representación». Pero además, y en segundo lugar, el Senado se concebirá igualmente como una garantía de la conservación, por parte de los estados, de la autonomía que justifica su existencia: «En este orden de ideas, puede observarse que la igualdad de votos concedida a cada estado es, a la vez, el reconocimiento constitucional de la parte de soberanía que conservan los estados individuales y un elemento para protegerla. Desde este punto de vista, la igualdad debería ser tan aceptable a los estados más extensos como a los más pequeños, ya que han de tener el mismo empeño en precaverse por todos los medios posibles contra la indebida consolidación de los estados en una república unitaria» ${ }^{9}$. El resumen, pues, no ofrece dudas, desde el punto de vista de la arquitectura constitucional: un Congreso formado por dos Cámaras, una (la de Representantes) predominantemente nacional, contrapesada por la otra (el Senado) predominantemente federal; y un Congreso contrapesado, a su vez, por una Presidencia de carácter mixto, al tiempo nacional y federal. Tal es el sistema de equilibrios (checks and balances) que en lo relativo al dispositivo federal se prevé, según la mayoritaria opinión federalista, en el texto de la Constitución de Filadelfia.

Las que, en su estela, procedieron también a la creación de parlamentos bicamerales en los Estados federales actualmente existentes

\footnotetext{
${ }^{8}$ Cfr. El Federalista, México, Fondo de Cultura Económica, 1998, pp. 161-162 (todas las cursivas en el original).

${ }_{9}$ Ambas citas en El Federalista, cit., pp. 262-263 (todas las cursivas en el original).
} 


\section{SENADOS, Representación Política y RePRESENTACión SOCIAL}

en el mundo ${ }^{10}$ no siempre iban a reconocer, en todo caso, los dos elementos característicos del federalismo norteamericano originario en relación con la llamada cámara de representación territorial (igualdad de los estados federados y elección de sus miembros por los legislativos estatales), y ni siquiera el primero de los dos, que es el que hoy en día lo define, después de la aprobación en 1913 de la XVII enmienda a la Constitución, en virtud de la cual los senadores pasaron a ser elegidos directamente por el pueblo de cada uno de los estados de la Unión. El parlamento de Canadá se compone de dos cámaras (la de los Comunes y el Senado), constituyendo esta última probablemente la segunda cámara más peculiar de todas las existentes en los países que a continuación estudiaré: el Senado, cuyo estatuto se regula en los artículos 21 a 36 de la Constitución, se compone de 105 miembros, designados formalmente por el Gobernador general, aunque en realidad por el primer ministro del país, con carácter vitalicio limitado (no podrán superar los 75 años de edad) y con un reparto que no es estrictamente igualitario: a cada una de las provincias de Ontario y Quebec les corresponderán 24 senadores, otros 24 de asignarán en conjunto a las provincias marítimas (10 a Nueva Escocia, 10 a Nueva Brunswick y 4 a la Isla del Príncipe Eduardo), 24 también a las Provincias del Oeste (6 Manitoba, 6 la Columbia Británica, Saaskatchewan y 6 Alberta), 6 a Terranova y, por último, uno a Yukon, Nunavut y los Territorios del Noroeste. La Constitución australiana dispone en su artículo primero que el parlamento se compone de una Cámara de Representantes y un Senado (siguiendo exactamente, por tanto, la denominación norteamericana) y dedica al segundo los artículos 7 a 23. Formado por senadores elegidos por los electores de cada estado a través de sufragio directo, el Senado se compone de 12 senadores por cada uno de los seis estados federados, y cuatro más para los territorios continentales: dos para el de la Capital y dos para el Territorio del Norte.

Los senados de los países iberoamericanos con sistema federal responden también, en mayor o menor grado, a los principios del bicameralismo nacido en Norteamérica. El Senado argentino, regulado en

${ }^{10} \mathrm{Cfr}$. , al respecto, aparte de la siempre útil guía de Daniel J. Elazar (Edit), Federal systems of the world. A Handbook of Federal, Confederal and Auntonomy Arrangements, Exeter, Longman Group Limited, 2ª edición, 1994, el amplísimo estudio dirigido Jörg Luther, Paolo Passaglia y Rolando Tarchi para el Centro Studio sul Federalismo, A World of Second Chambers. Handbook for Constitutional Studies on Bicameralism, Milán, Guiffrè, 2006. Y, también, el interesante trabajo de Enoch Alberti, «La representación territorial», en Fundamentos, no 3 (2004), pp. 279-330. 
los artículos 54 a 62 de la Constitución, se compondrá de 72 senadores, tres por cada una de las 23 provincias y 3 por la ciudad de Buenos Aires, elegidos de forma directa y conjunta, a través de un sistema mayoritario que otorga dos asientos al partido más votados y el tercer senador en disputa al que le siga en número de votos. La Constitución mexicana regula la composición y forma de elección del Senado en su artículo 56, según el cual la Cámara de Senadores se integrará por 128 miembros, de los cuales se elegirán tres en cada uno de los 31 estados del país y en el distrito federal: dos según el principio de la votación mayoritaria relativa y uno más que se asignará a la primera minoría. En cuanto a los 32 senadores restantes, serán elegidos según el principio de representación proporcional, mediante el sistema de listas votadas en una sola circunscripción plurinominal nacional. Brasil, cuyo Congreso Nacional se compone también de una Cámara de los Diputados y un Senado Federal, regula la composición y forma de elección de este último en el artículo 46 de la Constitución: según él, la cámara territorial se compondrá de un total de 81 miembros, representantes de los 26 estados del país y del distrito federal elegidos directamente siguiendo el principio mayoritario, a razón de tres senadores por cada uno de ellos.

La pauta del bicameralismo federal se mantiene también, en fin, en los Estados europeos de tal naturaleza, por más que en su plasmación se produzcan, como seguidamente se verá, algunas variaciones relevantes. Rusia cuenta con una Asamblea Federal o Parlamento de la Federación Rusa, al que la Constitución proclama como su órgano representativo y legislativo (art. 94 de la Constitución). Compuesta por dos cámaras (el Consejo de la Federación y la Duma del Estado), la primera, de carácter territorial, se conforma, a partir de un principio estrictamente igualitario, por 166 miembros a razón de dos representantes designados, a través de un sistema en elección en segundo grado, por cada uno de los diferentes 83 sujeto de la federación: uno del órgano legislativo y otro del órgano ejecutivo del poder del Estado (art. 95). El parlamento suizo, que se denomina Asamblea Federal, y al que la Constitución considera, a reserva de los derechos del pueblo y los cantones, la autoridad suprema de la Confederación Helvética (art. 148), se compone de dos cámaras (el Consejo Nacional y el Consejo de los Estados), el segundo de los cuales se conforma con 46 diputados de los cantones, según una distribución que, nuevamente, no es estrictamente igualitaria: seis de ellos eligen cada uno un diputado y los 20 cantones restantes del país ${ }^{11}$ eligen dos (art. 150).

${ }^{11}$ Se trata de los cantones de Obwald, Nidwald, Basilea Ciudad, Basilea Campiña, Appenzell Rodas Exteriores y Appenzell Rodas Interiores. Esa división responde al hecho de que en realidad el país se conforma con 23 cantones, pero tres están 


\section{SENADOS, Representación Política y RePRESENTACión SOCIAL}

Ese mismo precepto determina que son los propios cantones los que dictan las reglas aplicables a la elección de sus diputados en el Consejo de los Estados, pero en la actualidad rige sin excepciones un sistema de elección popular directa, que es mayoritario en todos, salvo en el cantón de Jura en donde responde a la regla proporcional ${ }^{12}$. La peculiar composición del Senado del Estado belga, que se une a la Cámara de Representantes para conformar el Poder Legislativo, se explica por las particularidades de su sistema federal, el que no sólo se federan regiones (Valonia, Flandes y Bruselas) sino también comunidades (francesa, flamenca y alemana) y regiones lingüísticas (holandesa, francesa, alemana y bilingüe). El Senado se compone, así, según el artículo 67 de la Constitución, en primer lugar, por 40 senadores de elección popular directa: 25 elegidos por el colegio electoral neerlandés y 15 por el colegio electoral francés; forman también parte del Senado 21 miembros elegidos, mediante votación en segundo grado, por los parlamentos de las comunidades: 10 por el parlamento de la Comunidad Flamenca, 10 por el de la Comunidad Francesa y uno por el de la Comunidad germanófona; $\mathrm{y}$, finalmente, seis senadores designados, igualmente en segundo grado, por los elegidos popularmente por los 35 senadores flamencos de elección popular y 4, designados del mismo modo, por los 25 senadores francófonos de elección popular. Ello da un total de 71 senadores, pero a ese grupo de senadores democráticos, y como una clara reminiscencia de la época de vigencia monarquía constitucional, se añaden los senadores por derecho propio (tres en la actualidad) ${ }^{13}$, pertenecientes a la familia real, que, sin embargo, por tradición, ni son tenidos en cuenta para el cálculo del quórum ni participan en las votaciones. Si la particularidad belga en relación con el Senado proviene de su peculiar sistema de entidades federadas, la española tiene que ver, como es sabido, con la génesis del proceso descentralizador y las previsiones contenidas en una Constitución elaborada antes de ella y no reformada con posterioridad. Tal circunstancia explica que, a diferencia de todos los que han sido hasta ahora analizados, el Senado español, que el artículo 69 de la Constitución define, pese a todo, como la cámara de representación territorial, no lo

compuestos por semicantones (Basilea, Appenzell y Unterwald) lo que da lugar al número de 26 que se menciona habitualmente.

12 Remedios Sánchez Ferriz y María Vicenta García Soriano, Suiza. Sistema político y Constitución, Madrid. Centro de Estudios Políticos y Constitucionales, p. 113.

${ }^{13}$ Se trata de los príncipes Philippe, Astrid y Laurent de Bélgica, hijos del rey Alberto II y su esposa Paola Ruffo di Calabria, herederos, por ese mismo orden, a la Corona del país. 
sea en realidad básicamente de las entidades regionales federadas (las Comunidades Autónoma) sino de las provincias: según el precepto mencionado, cada una elige 4 senadores, además de los que corresponden a los archipiélagos canario y balear y a las Ciudades Autónomas de Ceuta y Melilla, lo que da un total de 208 senadores provinciales: 188 elegidos en las 47 provincias peninsulares, 16 en ambos archipiélagos y 4 en las Ciudades autónomas. Pero la Constitución añade a esos senadores provinciales, todos de elección directa a través de un sistema mayoritario de voto plural restringido, los designados por las Comunidades Autónomas: un senador para cada una de las 17 que conforman el Estado y otro más por cada millón de habitantes de su respectivo territorio, correspondiendo la designación, en este caso, a través de una votación en segundo grado, al respectivo parlamento regional ${ }^{14}$. El resultado de tales previsiones es que de los 264 senadores que componen actualmente en España la llamada cámara territorial, tal sólo 56 corresponde a las Comunidades Autónomas, siendo los restantes senadores provinciales, por más que, entre unos y otros, como pronto de verá, no exista, políticamente, a la postre ninguna diferencia.

La regulación austríaca parte de la disposición constitucional de que el Consejo Nacional y el Consejo Federal ejercen conjuntamente el poder legislativo de la federación (art. 24), y regula la composición y forma de elección del segundo de los dos a partir de dos principios esenciales, que determinan su naturaleza político-constitucional. Por un lado, y en lo que se refiere a la asignación de los escaños, los estados estarán representados en el Consejo federal en función del número de sus ciudadanos, de modo que el estado que tenga mayor número de ciudadanos enviará 12 miembros y cada uno de los demás estados tantos miembros como correspondan a la proporción entre el número de sus habitantes y la cifra total de población inicialmente tomada como referencia, pero con la particularidad de que se contarán como un número entero los restos cuando excedan la mitad de la cifra mencionada; en todo caso, cada

\footnotetext{
${ }^{14}$ Buena prueba de la peculiaridad española a la que se hace referencia en el texto reside en el hecho de que el aparado $5^{\circ}$ del artículo 69 de la Constitución, al referirse a la designación de los senadores autonómicos, la atribuya a la asamblea legislativa o, en su defecto, al órgano colegiado superior de la Comunidad Autónoma, redacción que supone una perfecta expresión de la incertidumbre que existía en el momento constituyente respecto del modelo territorial que iba a implantarse finalmente en España tras la puesta en vigor de unas previsiones constitucionales, susceptibles de interpretaciones muy diferentes. Me he referido con detalle a la cuestión en mi libro Nacionalidades históricas y regiones sin historia. A propósito de la obsesión ruritana, Madrid, Alianza Editorial, 2006, pp. 37-54.
} 
estado tendrá derecho a una representación mínima de tres miembros (art. 34). Siguiendo tales reglas, el reparto en la actualidad es el siguiente: 3 escaños (Burgerland y Vorarlberg), 4 (Carintia y Salzburgo), 5 (Tirol), 9 (Estiria), 11 (Baja Austria y Viena) y 12 (Alta Austria). El segundo de los principios antes citados es el relativo al sistema de elección de los miembros del Consejo Federal, elección que, según el artículo 35 de la Constitución, corresponderá, mediante voto indirecto, a las dietas regionales por toda la duración de su respectiva legislatura conforme al principio de la representación proporcional, si bien corregido con arreglo a los criterios que fija el propio artículo. Ello significa, entre otras cosas, que expirada la legislatura en una Dieta regional, los miembros enviados por ella al Consejo seguirán en funciones hasta que la nueva Dieta proceda a realizar la correspondiente elección.

El sistema alemán, con el que cerraré mi breve recorrido por los doce Estados objeto de este trabajo, presenta, sin duda, la mayor particularidad de todas las que han sido previamente analizadas. Y ello porque, según ha señalado con acierto Wilkfried Röhrich, su peculiarísimo Senado (Consejo Federal o Bundesrat) «más que como una segunda cámara funciona como un segundo Gobierno» ${ }^{15}$. Tal hecho, en cuyas manifestaciones pasaré a centrarme de inmediato, resulta, sin duda, incomprensible sin tener en cuenta sus antecedentes remotos, contenidos en la Constitución que dio lugar a la unidad alemana, es decir, en la del Imperio, aprobada en 1871. El poder legislativo del nuevo Estado alemán se ejercería, según sus previsiones, a través de dos Cámaras -el Consejo Federal (Bundesrat) y la Dieta imperial (Bundestag)- cuya mayoría resultaba necesaria para la aprobación de cualquier ley del Imperio. El Consejo Federal, en tanto que cámara de representación de los territorios confederados, se compondría, a tener de lo dispuesto en el artículo $6^{\circ}$ de la Constitución, de un representante por cada uno de los Estados de la confederación, que votarían en la cámara con sufragio ponderado según la distribución que la propia Constitución establecía: los 31 votos asignados a los cuatro estados principales de la Confederación (17 a Prusia, 6 a Baviera, 4 a Sajonia y 4 a Würtemberg) suponían la mayoría absoluta de los 51 votos del Consejo. La Constitución de 1871 especificaba, además, en el mismo precepto, que cada Estado podría designar para el Consejo federal tantos mandatarios como votos le correspondieran en su seno, pero con la obligación de que todos los

${ }_{15}$ Wilkfried Röhrich, Los sistemas políticos del mundo, Madrid, Alianza Editorial, 2001, p. 22. 
designados por un mismo territorio deberían votar en idéntico sentido ${ }^{16}$. La naturaleza de una cámara que, como es fácil de apreciar, resultaba tan diferente a todas las otras de su género existentes en la época, estará íntimamente ligada a la postre, al peculiar proceso de formación de la unidad de alemana, que ya había fracasado, durante la Revolución de 1848, pese a la adopción en 1849 por «parlamento de Francfort» de una Constitución para la Alemania unida. Pero lo que no pudieron los revolucionarios de 1848 lo hará posible la política imperialista de Bismarck que, «enfocada esencialmente hacia el engrandecimiento de Prusia y el desarrollo del poder de la casa de los Hohenzollern» ${ }^{17}$, se traducirá, tras años de esfuerzo en pro de la unidad, en la firma de sucesivos tratados entre Prusia y los Estados que acabarán formando parte de la Confederación. Un proceso ese que, bajo hegemonía prusiana, originará el nacimiento de un Imperio configurado, al fin, como «una alianza de los príncipes alemanes, apoyada en las armas prusianas y legitimada por el júbilo de una burguesía liberal, de orientación nacionalista, que, en 1848, había intentado crear -sin éxito- un Estado nacional sobre la base de la soberanía popular y los derechos humanos» ${ }^{18}$. Tal génesis estatal generó entre los que participaron en ella, como parecía inevitable, una desconfianza mutua de la que el tipo de Senado previsto en la Constitución de 1871 vendrá a constituir una manifestación sobresaliente. Y será de ahí de donde pasará, tres cuartos de siglo después, aunque en un contexto histórico del todo diferente, a la Ley Fundamental de Bonn.

Es suficiente describir algunas de sus previsiones constitucionales respecto al vigente Bundesrat (arts. 50 y 51) para entender el profundo sentido de la apuntada afirmación de Röhrich. El primero de los dos preceptos aludidos determina que los estados cooperan en la legislación y en la administración de la Federación y en los asuntos de la Unión Europea a través del Consejo Federal. Tras ello, el artículo 51 dispone que éste se compondrá de miembros de los Gobiernos regionales nombrados y depuestos por el Gobierno respectivo, miembros que podrán ser representados por otros componentes del Gobierno correspondiente. Es decir, el Consejo no es un órgano representativo de los estados y, en esa misma medida, expresivo de su pluralidad política interna, sino un

\footnotetext{
${ }^{16}$ El texto de la Constitución del Imperio, de 1871, puede consultarse en Archivio delle Costituizioni Storiche, elaborado por el Dipartimento de Scienze Giuridiche de la Università di Torino (http://www.dircost.unito.it/index.shtml).

${ }^{17}$ Jacques Droz, Alemania. La formación de la unidad alemana. 1789-1871, Barcelona, VicensVives, 1973, cit., p. 260.

${ }^{18}$ Hagen Schulze, Breve historia de Alemania, Madrid, Alianza Editorial, 2009, p. 129.
} 


\section{SENADOS, Representación Política y RePRESENTACión SOCIAL}

órgano en que los propios estados estarán presentes por sus ejecutivos respectivos. La Constitución, añade, además, que esa presencia no será igualitaria, sino proporcional al peso demográfico de los estados federados, que votarán en el Consejo según la ponderación que determina el texto constitucional: cada estado uno tendrá, como mínimo, tres votos; los de más de dos millones de habitantes, cuatro; los de más de seis millones, cinco y seis, en fin, los de más de siete millones, lo que da en la actualidad un total de 69 votos en la cámara ${ }^{19}$. Finalmente, para completar sus peculiaridades, y en coherencia con todo lo anterior (carácter no representativo de los consejeros y voto ponderado por población) la Constitución determina que los votos de cada estado, que podrá enviar tantos miembros al Consejo Federal como votos tenga, sólo podrán emitirse en bloque y por los miembros presentes o sus representantes.

\section{PERO, ¿SON TERRITORIALES LOS ACTUALES SENADOS FEDERALES?}

He dejado para el final, con toda la intención, los modelos senatoriales alemán y austriaco no sólo porque, entre los europeos, guardan entre sí un relativo parecido, sino además, porque sus caracteres me van a servir como entrada inmejorable para abrir el tratamiento de la cuestión que ahora nos importa: la de si puede afirmarse, en el pleno sentido de ese término, que los senados federales (valga la expresión para designar sus denominaciones diferentes) son cámaras de representación territorial o, lo que es lo mismo, que lo son por el hecho de que representan a los territorios de un modo sustancialmente diferente a como lo hacen las llamadas cámaras bajas o de representación popular. Dicho de otro modo: aún partiendo del hecho de que «toda representación tiene una base territorial, y ninguna es propiamente territorial, en sentido estricto», la utilización del concepto de representación territorial haría referencia «al fenómeno por el cual se hacen presentes en los mecanismos de decisión del Estado unas instancias, con base poblacional o ciudadanía propia, a las que se reconoce entidad y poder político, que son algo distinto y diferenciado de la instancia general, del pueblo federal, del todo en el que

\footnotetext{
${ }_{19}$ Poseen seis votos los estados de Baden-Württemberg, Baviera, Baja Sajonia y Renania del Norte-Westfalia; cinco, Hesse; cuatro, Berlín, Brandenburgo, Renania-Palatinado, Sajonia, Sajonia-Anhalt, Schleswig-Holstein y Turingia; y, finalmente, tres, Bremen, Hamburgo, Mecklemburgo-Pomerania Occidental y Sarre.
} 
se integran». La cuestión es, sin embargo, la de saber si, como ha sido señalado, «la presencia de las partes (de sus pueblos, o de alguna de las instituciones que las representan) mediante una representación específica (que es distinta de la de sus ciudadanos individuales en el conjunto) rompe la lógica meramente aritmética de la composición del pueblo federal (equivalente a la suma de todos los ciudadanos federales) e introduce un factor de corrección, un contrapeso que permite que otros sujetos y otros intereses estén presentes también en el proceso de formación de la voluntad federal $»^{20}$. Con ello no quiere hacerse referencia, claro está, al hecho de que la conformación político-partidista de los senados sea más o menos diferente de la de las cámaras del pueblo, pues es obvio que diferentes composiciones y formas de elección tenderán siempre, por definición, a traducirse en que existan tales diferencias y a que, en algunos casos, sean incluso muy notables. Lejos de ello a lo que aludo al preguntarme por la autenticidad de esa llamada representación territorial es al hecho de si los senados representan una voluntad de los territorios que puede llegar a ser diferente de la de los habitantes de los territorios cuando estos se unen al conjunto de los que componen el Estado para la elección de las cámaras de representación popular. La respuesta a esta cuestión, central desde mi punto de vista para afirmar la existencia en los estados federales de una característica de la que carecerían los Estados unitarios con parlamentos también bicamerales, exigirá primero que nada hacer un balance sumario de lo analizado previamente, para poder fijar luego alguna regla de carácter general. Comenzaré, pues, por el balance, que ha de ir referido a tres aspectos esenciales: el carácter o no igualitario de le representación en los senados de las entidades federadas, la naturaleza directa o indirecta de la elección de los miembros que en cada caso los componen y la libertad o no del mandato que reciben de quienes (directa o indirectamente) han procedido a su elección.

a)La primera dimensión presenta una indudable relevancia, pues el factor de corrección y contrapeso que los senados pueden suponer respecto a la representación que expresan las otras cámaras de los respectivos parlamentos nacionales «es tanto mayor cuanta más igualdad existe en la representación de las diversas instancias territoriales, es decir, cuanto más independiente sea el peso de su representación en la escasa

${ }^{20}$ Cfr. Enoch Alberti, «La representación territorial», en Fundamentos, cit., pp. 281-282 y 286, respectivamente; $y$, en general, con consideraciones de mucho interés sobre la naturaleza jurídico-política de la representación territorial, pp. 279-296. 


\section{SENADOS, Representación Política y RePRESENTACión SOCIAL}

federal del peso de su población en el conjunto» ${ }^{21}$. No es casual, en tal sentido, que, según antes se apuntaba, fuera éste justamente el motivo de discusión fundamental cuando, con motivo del debate constituyente de Filadelfia, se procedió al diseño constitucional del senado del primer Estado federal de nuestra historia ${ }^{22}$. Pues bien, la conclusión en esta esfera es que de los doce Estados cuyos senados han sido analizados, la mayoría introducen el principio de igualdad en la representación de las entidades federadas en la segunda cámara del parlamento nacional: lo hacen así Estados Unidos (dos senadores por estado), Australia (12 senadores por estado), Argentina (tres senadores por provincia), Brasil (tres senadores por estado) y Rusia (dos representantes por cada sujeto de la Federación). En dos países más rige un sistema parcialmente igualitario: en México (tres senadores por estado, más 32 elegidos en un distrito único nacional plurinomial) y Suiza (seis cantones eligen cada uno un diputado y los 20 cantones restantes del país eligen dos). No rige, en fin, el criterio igualitario en Canadá, Austria, España (para los senadores autonómicos, aunque sí rige para los senadores provinciales, que no autonómicos) Bélgica y Alemania, países todos en donde se aplican diversos criterios corregidos de proporcionalidad.

b) La significación del segundo de los criterios apuntados -la elección de los miembros de las segundas cámaras por voto popular directo o a través de una designación, en segundo grado, por uno o más órganos de las entidades federadas- está directamente relacionada, según es fácil de entender, con el último criterio (la libertad de juicio político o el mandato cerrado de esos miembros) por dos razones: en primer lugar, porque, al menos en teoría, resulta más factible concebir un mandato cerrado (es decir, con instrucciones) allí donde la elección es indirecta y procede de una institución previamente elegida, cuya posición política resulta posible fijar, en consecuencia, a través de la expresión de la voluntad de la mayoría de quienes la componen; y en segundo lugar porque, en cualquier caso, allí donde el mandato es libre -es decir, donde sea cual sea la fuente de la designación, los nombrados no quedan vinculados a las instrucciones que reciben de quienes han procedido a su elecciónresulta mucho menos relevante cual sea el sistema de designación de los miembros del Senado. En Estados Unidos, donde rigió durante más de una centuria el sistema indirecto de elección de los senadores por los parlamentos estatales (entre 1787 y 1913 en que se aprobó la XVII

${ }^{21}$ Ibidem, p. 286

${ }^{22}$ William H. Riker, The Development of the American Federalism, cit., pp. 30-32. 
enmienda que estableció la elección de aquellos por el pueblo de los estados de la Unión) lo cierto es que algunas asambleas estatales transmitían instrucciones a sus propios senadores, pero sin que el incumplimiento de ellas por parte de los senadores estatales tuviera mayores consecuencias, dado que en ningún momento llegó a disponerse un sistema de recall (revocación del mandato o, lo que es lo mismo, retirada de la confianza con la obligación de dimitir ${ }^{23}$ para desposeer de su cargo a los representantes que hubieran violado el mandato eventualmente recibido, de modo que en «ausencia de recall la sanción más evidente era el rechazo de la reelección ${ }^{24}$. En realidad, la experiencia histórica del Senado estadounidense pone de relieve que sus miembros se fueron independizando progresivamente del mandato de los parlamentos estatales ya antes de la desvinculación final producida tras la aprobación de la XVII enmienda, que significó la culminación de la nacionalización de la cámara, y demuestra también que, en todo caso, la vinculación entre los senadores y quienes los elegían en segundo grado en los parlamentos estatales tendió a ser siempre bastante tenue ${ }^{25}$. Fuere como fuere, el ejemplo norteamericano iba a cundir en esta esfera en menor medida que en la previamente analizada, pues en lo referido a los sistemas de elección directa o indirecta de los miembros de las segundas cámaras lo cierto es que la diversidad resulta muchísimo menor. En efecto, tras el abandono del sistema por Estados Unidos en 1913, y dejando de lado el peculiar caso canadiense, donde los senadores son designados por el primer ministro del país, tan sólo tres países de los analizados establecen una elección en segundo grado de los miembros de la Cámara de representación territorial: Rusia (donde uno de los dos representantes estatales en el Consejo de la Federación es nombrado por el ejecutivo y otro por el legislativo de la unidad territorial de que se

\footnotetext{
${ }^{23}$ Véase, respecto de la institución, que existe en la actualidad en algunos Estados iberoamericanos, en su variante de recall popular (Perú, Venezuela, Colombia, Ecuador o Bolivia) y se prevé también en diversas Constituciones estatales de países federales (se introdujo, por ejemplo, en 15 Constituciones estatales de los Estados Unidos entre 1908 y 1979), Roberto Scarciglia, Il divieto di mandato imperativo. Contributo a uno studio di diritto comparato, Padua, Cedan, 2005; Beatriz Tomás Mallén, Transfuguismo parlamentario y democracia de partidos, Madrid, Centro de Estudios Políticos y Constitucionales, 2002 y Thoma E. Cronin, Direct democracy: the politics of initiative, referendum and recall, Harvard University Press, 1989.

${ }^{24}$ Enoch Alberti, «La representación territorial», en Fundamentos, cit., p. 300 (nota 17) y, con gran detalle, William H. Riker, The Development of the American Federalism, cit., pp. 141 , de donde procede la cita textual.

${ }^{25}$ Cfr. William H. Riker, The Development of the American Federalism, cit., pp. 139 y ss.
} 


\section{SENADOS, Representación Política y RePRESENTACión SOCIAL}

trate), Austria (donde los miembros del Consejo Federal son designados por las dietas regionales) y Alemania, donde el Bundesrat forma su voluntad con los votos que corresponden a los diferentes estados federados. Bélgica y España presentan, por último, sistemas de elección que, en sentido estricto, deberían ser calificados como mixtos: en Bélgica, porque una parte de los senadores elegidos por voto directo proceden a su vez a designar a otros miembros de la cámara, mediante un sistema de elección en segundo grado; y en España, porque junto a un bloque de senadores provinciales, de elección directa, aunque no por los territorios autónomos, existe otro, de tamaño mucho menor, en el que la elección se lleva a cabo por los parlamentos autonómicos.

c) Poco hay que decir, en fin, en relación con cómo se reparte por países la tercera variable -la relativa a la naturaleza libre o instruida del mandato- tras lo que ya acaba de apuntarse en tal sentido ${ }^{26}$. Y es que, salvo en Alemania, donde los miembros del Bundesrat votan según las instrucciones que reciben de los ejecutivos estatales a los que representan, en todos los demás países estudiados $-\mathrm{y}$, en general, en todos los Estados federales-, los miembros de la cámara territorial no tienen en la actualidad más limitaciones en su libertad de voto que las que condicionan a sus colegas de la cámara de naturaleza popular: las derivadas del mandato de partido al que todos están sujetos con mayor o menor grado de rigor. Tal constatación incorpora, en todo caso, dos motivos de reflexión que me permitirán acercarme a la formulación de la regla general a las que previamente se hacía referencia. El primero es que sólo donde el voto de los miembros de la cámara territorial está instruido existe la posibilidad de que, de esa propia instrucción, se deduzca de un modo natural la característica que permite hablar en relación con los senados de una auténtica representación territorial: me refiero, obviamente, a la naturaleza unitaria del voto de la unidad federada de que se trate en cada caso. A fin de cuentas, no es en absoluto casual que también sea Alemania (el único sistema federal en el que el voto de su senado es instruido) el único igualmente en el que se ha constitucionalizado la exigencia de que el voto de los Länder se emita de un modo unitario: el artículo 51.3 de su Constitución dispone, como ya

\footnotetext{
${ }^{26}$ Análisis conjuntos de las variables citadas en el texto, en los que se incluyen tablas de gran utilidad, pueden verse en el trabajo ya citado de Enoch Alberti, «La representación territorial», en Fundamentos, cit., pp. 296 y ss. y cuadro 4; y en Ronald L. Watts, Sistemas federales comparados, Madrid, Marcial Pons, 2006, pp. 213-219 y tablas 16 y 17.
} 
vimos, que los votos de un estado sólo se podrán emitir en bloque y por los miembros presentes o sus representantes: «Esta es precisamente la consecuencia natural de la instrucción de voto: su emisión en términos unitarios $[\ldots]{ }^{27}$. Tal es la razón por la que cabe afirmar que cuando los representantes de los estados alemanes de Hamburgo, Hesse o Bremen (y son sólo, como es obvio, tres ejemplos tomados al azar) manifiestan la voluntad de sus respectivos territorios, esa afirmación resulta verdadera en un sentido en que no lo es en modo alguno cuando la hacemos en relación con los representantes que forman parte de los entes territoriales de cualquier otro estado federal. Y ello porque, en estos últimos, la voluntad que los entes federados manifiestan en la cámara territorial o expresa la pluralidad política de sus representantes (y, por tanto, no es una voluntad unitaria sino varias, tantas como partidos representen al territorio de que se trate en cada caso en el senado) o es, en última instancia, el producto resultante de decidir, con arreglo al criterio de la mayoría, entre las diversas posiciones en disputa: en suma, y por decirlo de una vez, los miembros del estado de Bremen en el Bunderat expresan una posición, pero los dos senadores de California no tienen por qué hacerlo si uno pertenece al Partido Demócrata y otro al Republicano, como no lo hacen, por idénticas razones, los miembros de las diputaciones territoriales, políticamente plurales, de cualquier otro Estado federal. Y es que, a la postre, el Bundesrat alemán no es una cámara representativa en el sentido en el que, con la excepción de la canadiense, lo son las ya estudiadas: en él no se representa la voluntad plural de los electores, sea cual sea la base territorial de su representación, sino que se manifiesta la posición unitaria de los Gobiernos de los Länder, lo que resulta por completo diferente.

Ello nos lleva ya, como de la mano, a la segunda de las cuestiones apuntadas, dado que la determinación del papel que juegan los senados en los modernos Estado federales no puede dejar en un plano secundario un hecho primordial: que todos esos Estados son, aunque no en idéntico grado, Estados de partidos ${ }^{28}$, en el sentido que solemos otorgar a dicho término, es decir, Estados en los que la representación política -con independencia de la base, popular o territorial, sobre la que aquella se construye- es monopolizada o casi monopolizada por los partidos políticos, que fijan mandatos generales a sus miembros en las cámaras, al margen de que, en los sistemas bicamerales, pertenezcan a una o a la otra. Es obvio, por supuesto, que la eficacia de ese mandato de partido

\footnotetext{
27 Enoch Alberti, «La representación territorial», en Fundamentos, cit., p. 313.

${ }^{28}$ Cfr., por todos, Manuel García Pelayo, El Estado de partidos, Madrid, Alianza Editorial, 1984.
} 


\section{SENADOS, Representación Política y RePRESENTACión SOCIAL}

dependerá de la capacidad de las organizaciones políticas para disciplinar el voto de sus representantes en las cámaras del parlamento nacional; y lo es que la experiencia demuestra que tal disciplina resulta, de hecho, mayor o menor, dependiendo de los casos. Sin embargo, aún siendo cierto que la referida disciplina ha solido estar más relajada en los sistemas presidenciales que en los parlamentarios, en todos la tendencia general se ha dirigido últimamente en el sentido de su fortalecimiento progresivo ${ }^{29}$. Por otro lado, la eficacia del mandato de partido como elemento disciplinador del voto de los parlamentarios nacionales (de una u otra cámara) ha dependido también de la homogeneidad del sistema de partidos en el conjunto del territorio del Estado o, lo que es lo mismo, del grado de implantación general de los grandes partidos nacionales, de modo que allí donde existen fuertes partidos nacionalistas -o, más allá de esa ideología, importantes partidos de ámbito no estatal, que responden a otro tipo de cleavages o líneas sociales de fractura- la conformación política interna de las cámaras puede cambiar de forma sustancial, con efectos muy notables sobre la dinámica de funcionamiento general del sistema federal: «La existencia de sistemas de partidos integrados supone una condición para un federalismo estable, en el cual los sujetos federados constituyen parte integrante del gobierno nacional (Estados Unidos y Alemania). Los sistemas de partidos no integrados (como sucede en el caso de Canadá) favorecen, por el contrario federalismos menos estables, en los que la autonomía de los participantes y la propia Constitución nacional están constantemente sujetos a renegociación» ${ }^{30}$.

\section{IV. ¿CÓMO MEDIR LA TERRITORIALIDAD DE LOS PRESUNTOS SENADOS FEDERALES?}

En conclusión -y estas serían las reglas generales anunciadas-, la configuración final de los senados, en el sentido genérico que aquí viene

\footnotetext{
${ }^{29}$ Así lo señalaba ya hace años Daniel J. Elazar para el caso de los Estados Unidos, que pasa por ser el prototipo de un país con partidos poco estructurados y con laxa disciplina interna, cuando apuntaba que allí se manifestaba, desde finales de los años cincuenta «una creciente tendencia a construir partidos políticos nacionales, fuertes y centralizados. Esta tendencia se manifestó en los dos grandes partidos a lo largo de los sesenta y tuvo un relativo éxito», en Exploración del federalismo, Barcelona, Hacer Editorial, 1990, p. 133.

${ }^{30}$ Sofia Ventura (Edit.), Da Stato unitario a Stato federale. Territorializzazione della politica, devoluzione e adattamento istituzionale en Europa, Bolonia, Il Mulino, 2008, p. 24.
} 
dándosele a este término, como verdaderas cámaras de representación territorial, depende tanto de su diseño jurídico (constitucional y/o legal) como de factores estrictamente políticos ligados a la estructura y funcionamiento de los sistemas de partidos vigentes en cada país en diferentes momentos de su historia. En relación con su diseño, hay tres factores que influyen en la territorialidad del senado, aunque, como de inmediato se verá, el peso correlativo de cada uno de ellos no sea equiparable ${ }^{31}$. En primer lugar, la naturaleza igualitaria de la presencia en él senado de las diferentes unidades federadas, de modo que la territorialidad aumenta a medida que lo hace la igualdad y disminuye, hasta desaparecer, cuando aquella igualdad se reduce o deja de existir y es sustituida por el criterio de la representación más o menos proporcional a la distribución de la población: desde este punto de vista el Senado de Estados Unidos o el de Australia están mucho más territorializados que los Bundesrat de Austria o Alemania.

El segundo factor es el relativo a la forma de elección, directa o indirecta, de los miembros del senado, que convierte, en principio, en senados más territorializados a aquellos que son elegidos de forma indirecta por los órganos, generalmente parlamentarios, de las unidades federadas y menos a aquellos cuyos miembros son elegidos directamente por el pueblo: ello determina que la situación según este criterio pueda llegar a ser justamente la contraria que la que se deriva del primero, según lo demuestran los mismos ejemplos previamente utilizados: los Bundesrat de Austria y Alemania estarían, así, más territorializados que los senados de Estados Unidos y Australia.

Pero ni uno ni otro elemento implican en última instancia y de verdad, a mi juicio, el carácter territorial de las segundas cámaras, pues sean como sean elegidas (en primer grado o en segundo) y se configuren o no con un criterio igualitario, la existencia de un sistema de partidos más o menos disciplinado y uniforme $-\mathrm{y}$ todos lo son en la actualidad de un

31 Enoch Alberti realiza en su estudio un ensayo de medición cuantitativa de la representatividad territorial de las segundas cámaras teniendo en cuenta cinco criterios (reparto escaños/votos, posición respecto de la legislación, posición en la reforma constitucional, composición [representación específica] y tipo de mandato [libre o instruido]) a los que otorgan de menor a mayor representatividad una valoración de uno a cinco: el resultado final es que, en orden de más a menos representatividad, los senados de los que aquí me ocupo se ordenarían del siguiente modo: Bundesrat alemán (21 puntos sobre un total de 25), Senado estadounidense, Ständerat suizo y Senado australiano (19), Bundesrat austriaco (15), senados belga y canadiense (13) y Senado español (12). En «La representación territorial», en Fundamentos, cit., p. 314 y cuadro 7. 


\section{SENADOS, Representación Política y RePRESENTACión SOCIAL}

modo $\mathrm{u}$ otro en los países estudiados- produce como efecto que los senados manifiesten una voluntad que, formada a través del criterio democrático mediante las oportunas votaciones llevadas a cabo en su interior, será a la postre y de forma idéntica a lo que acontece en las cámaras bajas, la de los partidos que tienen en ellos mayoría. Que esas voluntades sean o no similares, medidas en términos de correlación de fuerzas políticas entre mayoría y minorías, a las existentes en las cámaras bajas $^{32}$ puede ser, desde luego, muy relevante desde el punto de vista del funcionamiento del régimen político: y así, mientras «en los sistemas presidenciales -como en los Estados Unidos- mayorías diversas en la Cámara de representantes y en el Senado ciertamente plantean una complicación, pero es una dificultad que el presidencialismo puede superar y esta acostumbrado a superar», la situación resulta diferente «en el contexto de los sistemas parlamentarios», donde, según lo ha subrayado Sartori, «el problema es mucho más grave»33. Más allá de ello, lo cierto es, en cualquier caso, que ese posible contraste de voluntades entre ambas cámaras no afecta al carácter territorial de las segundas, que lo serán o no con independencia de que la conformación de su voluntad política coincida o no con la de las cámaras bajas y facilite o no la dirección política que impriman las mayorías parlamentarias existentes en ellas.

Por eso, desde mi punto de vista, el criterio auténticamente relevante es el tercero, es decir, el que se deduce de la libertad o no de voto de los miembros que en la segunda cámara de que se trate en cada caso representan a las diferentes unidades federadas: de este modo, si el voto no está instruido ni, en consecuencia, es unitario -lo que, según hemos visto, acontece en todas partes, con la única excepción de Alemania- el papel de las segundas cámaras sólo se distingue del de las primeras de forma accidental: es decir, pueden distinguirse o no según sea su conformación política concreta. Pero, si como sucede en el Bundesrat alemán, los miembros de la cámara actúan, sirviéndose de los votos que cada delegación estatal tiene atribuidos, según las instrucciones que reciben del correspondiente Gobierno regional, a través de una única manifestación de voluntad por cada asunto y para cada uno de los Länder, no cabe la menor duda de que la diferencia entre la primera cámara y la

32 Contiene, por ejemplo, datos en ese sentido, para Alemania y Estados Unidos, el estudio de Enoch Alberti, «La representación territorial», en Fundamentos, cit., pp. 292295 y cuadros 2 y 3

33 Giovanni Sartori, Ingeniería constitucional comparada. Una investigación de estructuras, incentivos y resultados, México, Fondo de Cultura Económica, 1994, p. 201. 
segunda es sustantiva, porque esa diferencia reside precisamente en aquello que convierte a una asamblea en una genuina cámara de representación territorial: que la posición que en su seno expresa cada unidad federada es la del correspondiente territorio en un sentido estricto, es decir, en un sentido que no se produce en ninguno otro de los senados federales estudiados. Daniel Elazar lo reconocía así, con toda claridad, en su Exploración del federalismo, cuando apuntaba que «tal vez la segunda cámara más eficaz sea el Bundesrat alemán, que actúa como portavoz de los Länder» tanto en lo referente a la protección de sus intereses como en lo relativo a su protagonismo en el quehacer federal. En cambio, añadía el profesor norteamericano, «el senado de los Estados Unidos es una de las cámaras federales de menor eficacia, pues sus miembros son elegidos ad personam y no se requiere de ellos que defiendan su respectivo estado per ser. El senado canadiense, concluía, es decorativo y ni tan sólo pretende realizar una función federalista» ${ }^{34}$. Del mismo modo, Sartori subraya, en referencia al Bundesrat, que «es Alemania, me parece, la que muestra la forma en que puede incluirse el bicameralismo federal en un sistema parlamentario» ${ }^{35}$.

¿Quiere esto decir que el Bundesrat alemán es la única auténtica cámara de representación territorial que existe en el mundo federal? No exactamente: significa que las demás no lo son, ni de lejos, en el sentido en que lo es el Bundesrat, lo que explica, por ejemplo, que cuando en algún país (en España, por ejemplo) se ha debatido largo y tendido sobre la posibilidad (o la necesidad) de territorializar de forma efectiva un senado que a la postre no lo es se haya planteado por algunos especialistas la conveniencia de acudir al modelo alemán antes que a ninguno de los existentes en el derecho comparado ${ }^{36}$. Es esta constatación la que explica algo que de otro modo podría resultar más que llamativo: que en el presente análisis no se haya hecho todavía referencia a las funciones que las segundas cámaras tienen atribuidas en los Estados federales ${ }^{37}$. La

${ }^{34}$ Daniel J. Elazar, Exploración del federalismo, cit., p. 232.

35 Giovanni Sartori, Ingeniería constitucional comparada. Una investigación de estructuras, incentivos y resultados, cit., p. 204.

${ }^{36}$ Cfr. Eliseo Aja. El Estado autonómico. Federalismo y hechos diferenciales, Madrid, Alianza Editorial, 2003, p. 265. Para una propuesta alternativa, que trata de conseguir resultados parciales similares, pero manteniendo un Senado representativo, puede verse mi libro Nacionalidades históricas y regiones sin historia. A propósito de la obsesión ruritana, cit., pp. 200-215.

37 Cfr., al respecto, Enoch Alberti, «La representación territorial», en Fundamentos, cit., pp. 307-309 y cuadro 5, y Ronald L. Watts, Sistemas federales comparados, cit., pp. 218219 y tablas 16 y 17, obras que, en conjunto, contienen información detallada al 


\section{SENADOS, Representación Política y RePRESENTACión SOCIAL}

razón de tal ausencia tiene que ver, ciertamente, con nuestro punto de partida, es decir, con la idea de que lo que define la naturaleza territorial de una asamblea son precisamente los elementos aludidos y no las funciones que pueda tener atribuidas, pues aunque tales funciones tengan en teoría, en ciertos casos, un claro impacto federalizante (porque se refieran, por ejemplo al sistema de financiación, o a las reformas constitucionales que afecten al diseño del modelo federal), tal impacto deja sencillamente de tener ese carácter para pasar a tener otro sustantivamente diferente cuando la cámara alta, por ser una cámara primordial o exclusivamente vertebrada sobre los partidos políticos, se convierte en realidad en una mera reproducción, en términos políticos, de la cámara baja, y ello al margen de que sea o no coincidente la correlación de fuerzas existente en la una y en la otra. Por decirlo de otro modo, lo que convierte en territorial, por ejemplo, al eventual veto del que pueda disponer la cámara alta respecto de la baja no es el hecho mismo de que exista tal posibilidad de vetar, ni siquiera las materias a las que ese veto pueda referirse, sino la circunstancia de que el veto sea la expresión de la posición de las unidades federadas y no del cuerpo electoral de la nación que, debido al sistema de composición del senado o a su sistema de elección, sencillamente se expresa de un modo distinto desde el punto de vista de la correlación de fuerzas partidistas de cómo se expresa en la cámara que, supuestamente en exclusiva, representa al cuerpo electoral. $\mathrm{Y}$ es que, como ha sido señalado con acierto, en cualquier tipo de elección, «el sujeto representado es siempre una comunidad de ciudadanos, nunca un territorio, desprovisto o con independencia del pueblo que en él se asienta» ${ }^{38}$. Tan es así, que sólo cuando, al margen de cómo se conforme y de cómo se la elija, cambia de un modo sustancial la forma en que se expresa políticamente la representación de esa comunidad de ciudadanos en uno u otro tipo de

respecto sobre todos los países que tratamos, salvo la Federación Rusa y los tres Estados Iberoamericanos. Para Rusia véase Manuel García Álvarez, «Rusia», en Carlos Flores Juberías (Edit.), Las nuevas instituciones políticas de la Europa oriental, Madrid, Centro de Estudios Políticos y Constitucionales 1997, p. 646. Y para Argentina, Brasil y México los capítulos correspondientes de la obra de Domingo García Belaunde, Francisco Fernández Segado y Rubén Hernández Valle (Edits.), Los sistemas constitucionales iberoamericanos, Madrid, Dykinson, 1992, pp. 66-70 (Argentina), 147-150 (Brasil) y 585-588 (México). Las previsiones de las diversos Constituciones respecto de las facultades de las dos cámaras parlamentarias pueden consultarse en los textos según lo apuntado en supra nota 10.

38 Enoch Alberti, «La representación territorial», en Fundamentos, cit., p. 279. 
senados - porque en uno lo hace según el principio democrático y el voto libre, no sujeto a otro mandato que el de partido y en otro, por el contrario, de forma unitaria e instruida- las facultades que se le atribuyen a los senados tienen un impacto de territorialización sobre las decisiones parlamentarias que puede ser muy diferente: apenas perceptible en el primer supuesto y manifiesto en el segundo: «La particular composición del Bundesrat alemán -ha escrito Ronald Watts- y el hecho de disponer de un veto absoluto sobre la totalidad de la legislación federal que ha de ser ejecutada por los Länder y que supone más del 60 por ciento de toda la legislación federal han sido los principales argumentos de dicha influencia ${ }^{39}$. Y también, cabría añadir, las razones principales para explicar la profunda reforma llevada a cabo en 2006 en el federalismo germano, cuando, tras algún intento fallido, finalmente se aceptó de forma muy mayoritaria que la extraordinaria extensión de ese mecanismo de veto, puesto, en el pleno sentido de la palabra, en manos de los estados federados, resultaba claramente disfuncional para la correcta dinámica de funcionamiento del federalismo alemán: de hecho, la reforma supuso, entre otras cosas, una reducción muy sustancial del poder de veto del Bundesrat que vio drásticamente reducido -en más de la mitad- el porcentaje de materias sobre las que su concurso es necesario para que pueda decidir en Berlín el Bundestagio.

Por lo demás, y con la excepción del Bundesrat germano en los términos a los que acaba de aludirse, las segundas cámaras, tienen en los Estados federales una posición institucional que oscila entre la de plena paridad con las cámaras bajas $\mathrm{y}$, donde no, de subordinación respecto a ellas. Giovanni Sartori lo ha expresado así con rotunda claridad: «No hay ningún caso, en el mundo actual, de una Cámara Alta que esté por encima de la Cámara baja», principio al que el politólogo italonorteamericano añade a otro en relación con los sistemas parlamentarios: «Lo primero que

\footnotetext{
${ }^{39}$ Ronald L. Watts, Sistemas federales comparados, cit., p. 218.

${ }^{40}$ Cfr., diagnosticando el problema, Thomas Darnstädt, La trampa del consenso, Madrid, Editorial Trotta, 2005, en especial, pp. 85 y ss. Explicando las propuestas de solución al respecto, Antonio Arroyo Gil, El federalismo alemán en la encrucijada. Sobre el intento de modernización del orden federativo en la República Federal de Alemania, Madrid, Centro de Estudios Políticos y Constitucionales, 2006, en especial, pp. 122 y ss. Y, del mismo autor, analizando el contenido de la reforma finalmente aprobada, La reforma constitucional del federalismo alemán. Estudio crítico de la $52^{a}$ ley de modificación de la Ley Fundamental de Bonn, de 28 de agosto de 2006, Barcelona, Institut d'Estudis Autonòmics, 2009. Por mi parte, me he referido al problema en un breve artículo, «El federalismo alemán y "el movimiento necesario de las cosas"», en Revista de Libros, no 120 (2008), pp. 27-30.
} 


\section{SENADOS, Representación Política y RePRESENTACión SOCIAL}

hay que observar es que siempre que el poder de las dos cámaras es desigual, la más débil es la Cámara Alta» ${ }^{41}$. También Ronal Watts ha insistido en esa debilidad característica de los senados en los regímenes políticos adscritos al parlamentarismo, poniendo de relieve que «esto ha planteado en ocasiones interrogantes en las federaciones con forma de gobierno parlamentaria sobre si sus segundas cámaras disponen de suficiente capacidad para canalizar la influencia regional en el proceso de toma de decisiones a nivel central. Esta cuestión se refuerza por la común fortalece de la disciplina de partido en dichas formaciones con forma de gobierno parlamentaria» ${ }^{42}$. Análisis pormenorizados de la relación de poder entre ambas cámaras en los Estados federales confirman plenamente esa doble afirmación: así, por ejemplo, lo ha demostrado Enoch Alberti ${ }^{43}$ para una buena parte de los Estados federales a los que aquí vengo refiriéndome. Agrupando en cinco categorías posibles la posición de la segunda cámara respecto de la primera en relación con la legislación, ninguno de los países considerados responde a los modelos extremos de superioridad absoluta de la primera o de superioridad de la segunda, incluyéndose todos ellos en alguna de las siguientes categorías intermedias: superioridad de la primera, previa conciliación (España), ciertos ámbitos de igualdad, junto a otros de superioridad de la primera cámara (Alemania, Austria y Bélgica) e igualdad general entre la primera y la segunda cámara (Suiza, Estados Unidos, Canadá y Australia). En relación, por su parte, con la facultad de la reforma constitucional todas las naciones apuntadas se sitúan en la categoría de igualdad general entre la primera y la segunda cámara, salvo Austria (ciertos ámbitos de igualdad, junto a otros de superioridad de la primera cámara) y Canadá (superioridad de la primera, previa conciliación), aunque el criterio básico de participación territorial en materia de reforma, si aquella pretende ser auténtica, pasa, a mi juicio por la participación directa en ella de las entidades federadas. Por cerrar el análisis con los casos no referidos previamente, en la Federación rusa la cámara alta está en una posición de claro segundo plano respecto de la baja en la esfera de la función legislativa, incluyéndose, dentro de las categorías antes citadas, en la superioridad de la primera, previa conciliación (arts. 105-106 de la Constitución). Pero ello no impide, de todos modos, que el Consejo de la

\footnotetext{
${ }^{41}$ Giovanni Sartori, Ingeniería constitucional comparada. Una investigación de estructuras, incentivos y resultados, cit., pp. 198-199.

42 Ronald L. Watts, Sistemas federales comparados, cit., p. 218.

43 Enoch Alberti, «La representación territorial», en Fundamentos, cit., pp. 301-304, con datos muy pormenorizados, y cuadros incluidos en las pp. 308 y 314.
} 
Federación tenga «asignadas funciones de gran importancia que pueden servir, entre otras cosas, para que el Presidente [de la Federación] si así lo estima oportuno, actúe marginando a la Duma si cuenta con el apoyo de la cámara alta, tanto más cuanto que [...] la Constitución no delimita con claridad el campo de actuación de la ley y del ukás presidencial» ${ }^{44}$. Del capítulo quinto (sec. I, tit.I) de la Constitución argentina relativo a la elaboración de las leyes se deduce una posición de plena paridad en el proceso legislativo, es decir, de igualdad general entre la primera y la segunda cámaras del Congreso, dado que ningún proyecto desechado totalmente por una de las cámaras podrá repetirse en las sesiones de aquel año y que, en caso de desacuerdo entre ambas, habrá de recurrirse a un mecanismo de conciliación en el que Cámara de Diputados y Senado gozan de idénticos poderes (art. 81). El modelo mexicano es diferente, pues, junto a facultades privativas de cada una de las cámaras-reguladas respectivamente en los artículos 74 y 76 de la Constitución para la Cámara de Diputados y el Senado- la mayor parte de aquellas corresponden a las dos conjuntamente (art. 73), lo que permite calificar al sistema, desde este punto de vista, como de equilibrio general entre ambas cámaras. La situación es en Brasil, en fin, similar a la que acaba de describirse muy someramente para México: la mayor extensión de las funciones de las dos cámaras parlamentarias corresponden al Congreso Nacional (art. 48 de la Constitución) y en mucha menor medida a la Cámara de Diputados (art. 51) y al Senado (art. 52), que tienen, por tanto, en términos generales, una posición de paridad. Tal posición depende, sobre todo, en la generalidad de los países en los que existen parlamentos con dos cámaras -y no sólo, desde luego, en los de naturaleza federal-, según se deduce con bastante claridad del estudio del profesor Alberti citado previamente, de la regulación del veto que pueden presentar las cámaras altas a lo decidido por las bajas. A ese respecto, Giovanni Sartori ha fijado una regla general de medición que resulta, a mi juicio, de suma utilidad: «Nuestra otra variable -poder igual-poder desigual- se presta asimismo a una fácil medición. Aquí el indicador sería que el poder de la Cámara Alta sea sólo el poder de retrasar [...] o bien el poder de veto. En este último caso el veto puede ser: a) absoluto, esto es, sin recurso; b) absoluto solo en ciertos campos reservados; c) posible de ser anulado por una mayoría cualificada de la Cámara Baja, y d) posible de ser anulado por mayoría simple. Esto último difícilmente equivale a un poder de veto, porque se debe suponer que una ley ya aprobada por la Cámara Baja cuenta con mayoría en ésta. Pero lo cierto es que las mayorías que puedan superar la oposición del

${ }^{44}$ Cfr. Manuel García Álvarez, «Rusia», en Carlos Flores Juberías (Edit.), Las nuevas instituciones políticas de la Europa oriental, cit., p. 646. 


\section{SENADOS, Representación Política y RePRESENTACión SOCIAL}

senado varían, y que cuanto más altas se exija que sean, tanto más efectivo será el poder de veto» ${ }^{45}$.

La sumaria descripción hasta aquí realizada sobre la función de los senados en el derecho comparado federal, referida básicamente al ejercicio de la potestad legislativa por sus parlamentos bicamerales, no puede llevarnos a olvidar, de todas formas, una línea de reflexión adicional con la que pondré ya punto final a estas reflexiones: que entre aquellas funciones se incluyen las relativas a las relaciones con los ejecutivos respectivos, lo que permite realizar dos generalizaciones, cada una referida a los sistemas parlamentarios y a los presidencialistas. En los primeros, como ya se analizó, son las cámaras bajas las que, con la excepción del peculiar caso de Suiza, proceden a la elección del jefe del Gobierno, órgano que, también con carácter general, es responsable ante esa cámara, que asume en esa doble condición un poder y un protagonismo del que carecen los senados. En los sistemas presidencialistas, por su parte, el modelo norteamericano ha determinado la extensión a otros países de dos facultades del Senado federal de los Estados Unidos que tienen una notable importancia en la esfera de las que se conforman en tales sistemas como unas muy diferentes relaciones entre el poder legislativo y el poder ejecutivo. De un lado, la consiste en juzgar al presidente de la República y, eventualmente, a otros altos cargos del Estado, a través de impeachment, mediante un procedimiento que, iniciado a impulso de la cámara baja, se resuelve en la cámara alta finalmente: esa facultad ha pasado de la Constitución de Estados Unidos (art. I, secciones 2 y 3) a las de México (arts. 108-114), Brasil (arts. 52.I y 85-86) y Argentina (arts. 53 y 59) ${ }^{46}$. De otro lado, la que se manifiesta en la ratificación por parte de las cámaras altas de los nombramientos presidenciales, previo control de los mismos por parte de los miembros de la propia cámara, en lo que se conoce en el constitucionalismo norteamericano como advice and consent of the Senate (consejo y consentimiento del Senado: art II, secc. 2), competencia que en términos similares atribuyen también a sus senados dos de las tres Constituciones federales iberoamericanas a las que acaba de hacerse referencia: México (art. 76.II) y Brasil (52.III y IV). No deseo terminar, de todos modos, sin añadir una consideración final: que, según lo demuestra el caso norteamericano, los dos tipos de importantes poderes senatoriales a los

45 Giovanni Sartori, Ingeniería constitucional comparada. Una investigación de estructuras, incentivos y resultados, cit., pp. 200-201 (cursivas en el original).

${ }^{46}$ Cfr. Mario D. Serrafero, «El "impeachment” en América Latina: Argentina, Brasil y Venezuela», en Revista de Estudios Políticos, no 92 81996), pp. 137 y ss. 
que acaba de hacerse referencia no pertenecen, en realidad, tanto a los estados, en tanto que territorios, como a los partidos que se asientan en la cámara alta del Congreso Norteamericano, lo que vale tanto para el caso del advice and consent ${ }^{47}$ como, por más que en una medida no exactamente coincidente, para el impeachment presidencial ${ }^{48}$. Ello supone, de algún

${ }^{47}$ Un dato es bastante significativo al respecto: que en la centuria transcurrida entre 1850 y 1950 tan sólo dos nominaciones de miembros del Gobierno fueron rechazadas. Cfr. Joseph P. Harris, The Advice and Consent of the Senate, University of California Press, 1953, pp. 379. Por lo demás ese mecanismo perdería una gran parte de su eficacia como instrumento de control en manos del Senado, ya no digamos como supuesto medio de control de los territorios de la Unión sobre la política nacional, a consecuencia de la introducción de prácticas y costumbres que produjeron el efecto de vaciarlo parcialmente de las finalidades que perseguían los Padres Fundadores y sustituirlo por un sistema de patronazgo de partido coherente con la practica del spoils system: entre ellas, y de manera muy especial, la extraordinaria extensión que acabó dándosele a un sistema inicialmente concebido para ser aplicado de modo restringido; $y$, en segundo lugar, la costumbre, generalizada poco a poco, de la llamada cortesía senatorial (senatorial courtesy) en virtud de la cual los miembros del Senado actuaban con el senador patrón para un determinado nombramiento como esperaban que sus colegas actuaran con ellos llegada la ocasión: no interfiriendo la ratificación de la propuesta. Es cierto que, frente a ello se fue imponiendo también la costumbre de que el presidente consultase con los senadores de su partido en cada estado sobre el nombramiento de los puestos federales de ese estado, costumbre que limitaba muy seriamente el poder del presidente pero le ofrecía a cambio seguridad parlamentaria, pero ello, en lugar de favorecer al territorio favorece al partido en cada territorio. Esa conclusión final del sistema era expuesta por Harris con bastante claridad: «La antigua costumbre que exige al presidente consultar a los senadores de su propio partido sobre la nominación de los empleados federales en sus estados, y, por regla general, aceptar las propuestas derivadas de las mismas, se basa en una tradición partidista y no en la Constitución [...] Esa costumbre, reforzada por la regla no escrita de la "cortesía senatorial", transfiere virtualmente la función de nominación de esos puestos del presidente a cada uno de los senadores de su partido». Cfr. Joseph P. Harris, Ibidem, p. 377. Me he referido con detalle a la cuestión en mi trabajo «Acción de gobierno, política de nombramientos y control parlamentario», en Documentación Administrativa, nㅡㄹ 246247 (1997), pp. 168-178

${ }^{48}$ En los dos único casos en que llegó a juzgarse a un presidente de Estados Unidos, pues el impeachment contra Richard Nixon se interrumpió tras su dimisión en 1974, la disciplina de partido se mantuvo siempre entre los partidarios del presidente, aunque no así entre sus adversarios políticos: el demócrata Andrew Johnson (1 $17^{\circ}$ presidente, entre 1865 y 1869) fue declarado no culpable en 1868 (35 senadores votaron a favor de la culpabilidad frente a 19 en contra, minoría de la que formaron parte 7 senadores 


\title{
SENAdOS, RePresentación Política y RePRESENTACIÓN
} SOCIAL

modo, un colofón que permite comprender mejor hasta qué punto lo territorial y lo político-partidista están inextricablemente unidos en unas cámaras, que con la excepción del Bundesrat alemán, interpretan, en última instancia, la voluntad territorialidad según aquella se construye a partir de la correlación de fuerzas partidistas. Y es que la voluntad política de un territorio, plural por su propia naturaleza en un sistema democrática, solo se transforma, al fin y al cabo, en auténtica voluntad territorial cuando se sintetiza a través de la reducción de esa voluntad plural en unitaria mediante la atribución de la voluntad territorial a la de mayoría del órgano plural o a la del Gobierno que de la misma es expresión.

\author{
SENADOS, REPRESENTACIÓN POLÍTICA Y REPRESENTACIÓN \\ TERRITORIAL: REFLEXIONES SOBRE UNA EXTENDIDA CONFUSIÓN \\ SENATE, POLITICAL REPRESENTATION AND TERRITORIAL \\ REPRESENTATION: REFLECTIONS ON EXTENDED CONFUSION
}

republicanos, lo que impidió alcanzar los dos tercios necesarios para la aprobación del impeachment); el demócrata Bill Clinton ( $42^{\circ}$ presidente, entre 1993 y 2001), por su parte, fue declarado, igualmente no culpable, en 1999, de los cargos de perjurio (por 55 votos en contra, diez de ellos republicano, contra 45 a favor, todos republicanos) y de obstrucción a la justicia (por 50 votos en contra, cinco de ellos republicanos, contra 45 a favor, todos republicanos). Véase, para los casos de Johnson y Nixon, Alfred H. Kelly, Winfred A. Harbison y Herman Belz, The American Constitution. Its origins and development, Nueva York, W. W. Norton \& Company, 1983, pp. 350-356 y 692-700, respectivamente. 